\title{
Aspirin-induced hepatotoxicity and anemia in children with acute rheumatic fever
}

\author{
Derya Altay $^{1 \oplus}$, Özge Pamukçu ${ }^{2 \odot}$, Ali Baykan ${ }^{2 \odot}$, Kazım Üzüm $^{2 \odot}$, Duran Arslan ${ }^{1 \odot}$ \\ Departments of ${ }^{1}$ Pediatric Gastroenterology, Hepatology and Nutrition and ${ }^{2}$ Pediatric Cardiology, Erciyes University Faculty of \\ Medicine, Kayseri, Turkey.
}

\begin{abstract}
Background. The aim of this study was to investigate the frequency of anemia and hepatotoxicity associated with aspirin use in patients with acute rheumatic fever.

Methods. Patients with acute rheumatic fever followed at Erciyes University, Faculty of Medicine, Department of Pediatric Cardiology between 2015-2018 were reviewed retrospectively.

Results. A total of 286 patients with acute rheumatic fever were analysed. Aspirin treatment was started in 53 of the 286 patients $(18.5 \%)$ due to arthritis. The mean age of the patients who used aspirin was $10.7 \pm 2.5$ years. Aspirin-induced hepatotoxicity developed in $9(17 \%)$ of the 53 patients. Naproxen or ibuprofen was given to these patients as an alternative to aspirin. No side effects occurred in patients receiving naproxen or ibuprofen. In addition, $30 \%$ of 53 patients were initially anemic. The mean duration of aspirin use in the hepatotoxic patients who had anemia was longer than patients without anemia $(\mathrm{p}=0.02)$.
\end{abstract}

Conclusions. Patients with acute rheumatic fever should be closely monitored for aspirin hepatotoxicity. When aspirin hepatotoxicity develops, naproxen or ibuprofen treatment can be used safely.

Key words: acute rheumatic fever, arthritis, aspirin, aminotransferase elevation.

Acute rheumatic fever is a chronic, inflammatory and systemic disease caused by group A beta hemolytic streptococcal infections in genetically predisposed individuals. The disease manifests as a result of varying rates of inflammation of the heart, joints and basal ganglia. Rheumatic heart disease is mentioned in the presence of cardiac involvement and it is very important because it is responsible for mortality. Our country is among the middle-high risk populations in terms of acute rheumatic fever and rheumatic heart disease. ${ }^{1}$ Therefore, early diagnosis and treatment of acute rheumatic fever are important for the prognosis of patients. Jones criteria were established in 1944 for acute rheumatic fever diagnosis and updated periodically. In the light of the latest updates in 2015, the importance

\footnotetext{
$凶$ Derya Altay

dr.deryaaltay@gmail.com
}

Received 14th August 2020, revised 23rd October 2020, accepted 15th November 2020. of subclinical carditis in the diagnosis of acute rheumatic fever was emphasized and differences in terms of minor findings were highlighted in low and high risk populations. ${ }^{2}$ Traditionally, the management of acute rheumatic fever is antibiotic therapy, anti-inflammatory therapy and resting. The aim of antibiotic use is the eradication of Streptococcal infection in tonsils. As an anti-inflammatory treatment, aspirin is used in arthritis, corticosteroid is used in carditis, and aspirin is added during the tapering of streoid therapy in carditis. Aspirin, a non-steroidal anti-inflammatory drug, reduces the synthesis of proinflammatory cytokines by inhibiting cyclooxygenase. Aspirin is often hepatotoxic when used in high doses. Aspirin, a direct, intrinsic hepatotoxin, causes hepatocellular hepatotoxicity. Besides the increase in alanine aminotransferase (ALT), bilirubin levels are generally in the normal range. The clinical picture of the patients is mostly mild and asymptomatic. Abnormalities 
in liver tests recover rapidly within days with discontinuation of aspirin. People with aspirin hepatotoxicity can safely use other nonsteroidal anti-inflammatory drugs. ${ }^{3}$ Naproxen treatment is an alternative treatment approach if intolerance develops during aspirin use. ${ }^{4}$ When hepatotoxicity develops as a major side effect of aspirin, alternative non-steroidal antiinflammatory drug or steroid therapy should be used.

The aim of this study was to evaluate the patients who developed aspirin-induced hepatotoxicity due to acute rheumatic fever and needed an alternative non-steroidal antiinflammatory drug use and to investigate the rate of hepatotoxicity of aspirin, which is an important step in the treatment of acute rheumatic fever. In addition, the association of aspirin hepatotoxicity and anemia was also investigated in the present study.

\section{Material and Methods}

The files of the patients who were followed with the diagnosis of acute rheumatic fever between 2015 and 2018 at Erciyes University Faculty of Medicine, Department of Pediatric Cardiology were reviewed retrospectively. Patient records included age, sex, presence of arthritis, aspirin use, aspirin related side effects, serum aminotransferase levels, hemoglobin values, C-reactive protein (CRP) and erythrocyte sedimentation rate (ESR), and non-steroid antiinflammatory treatments other than aspirin. Patients with isolated carditis or chorea were excluded.

In the Department of Pediatric Cardiology, acute rheumatic fever was diagnosed according to the modified Jones criteria. ${ }^{2}$ The first step in the anti-inflammatory treatment of arthritis in patients with acute rheumatic fever was aspirin treatment. If there was carditis together with arthritis, corticosteroid therapy (methyl prednisolone 1-2 mg / kg / day, maximum 48 $\mathrm{mg}$ / day) was given orally for two weeks. At the end of the treatment, the steroid dose was reduced and aspirin was added to the treatment. Steroid therapy was tapered in seven days. The biochemical evaluation of liver enzymes of the patients were performed before treatment. The dose of aspirin was started at a dose of 80-100 $\mathrm{mg} / \mathrm{kg} /$ day divided four times daily, after a full dose was given for two weeks, it was reduced and discontinued in two weeks. After aspirin treatment was started, patients were asked to come for a check-up of liver enzymes in the first week of the treatment and then, control of liver enzymes was done weekly. Patients were monitored for side effects such as aspirininduced hepatotoxicity and gastrointestinal bleeding. Elevated aminotransferase levels up to five times higher than normal was defined as mild, five to ten times higher than normal was defined as moderate and greater than ten times higher than normal limit after starting aspirin treatment was defined as severe aminotransferase elevations. ${ }^{5}$ The upper limit of normal for aspartate aminotransferase (AST) and ALT is $40 \mathrm{U} / \mathrm{L}$ in the Biochemistry Laboratory in our hospital. In patients with carditis and arthritis, aspirin was started at a dose of $75-80 \mathrm{mg} / \mathrm{kg} /$ day one week before steroid discontinuation and the treatment duration was four weeks. In patients with arthritis, aspirin was discontinued and other non-steroidal antiinflammatory drugs such as naproxen and ibuprofen were switched to aspirin. All patients with hepatotoxicity were evaluated for serum total and direct bilirubin, albumin, prothrombin time, INR, viral serology, ceruloplasmin and liver autoantibodies to rule out other causes of hepatitis. In the Biochemistry Laboratory in our hospital, the normal value for CRP was $<5 \mathrm{mg} / \mathrm{L}$ and the normal value for ESR was $<20 \mathrm{~mm} / \mathrm{h}$. Hemoglobin levels were $<11.6 \mathrm{~g} / \mathrm{dL}$ for children aged 8-12 years, $<11.8 \mathrm{~g} / \mathrm{dL}$ for girls aged 1215 years and $<12.3 \mathrm{~g} / \mathrm{dL}$ for boys aged 12-15 years were defined as hemoglobin levels were below -2SD and the patients were accepted as anemic. Anemia etiologies of the patients were investigated from the file records.

Ethics committee approval was received from Erciyes University Faculty of Medicine for this 
study (Date: 07.11.2018, Approval Number: 2018/573). Written and verbal consents were obtained from the parents of the patients.

\section{Statistical analysis}

In this study, the data were evaluated with descriptive statistics method (SPSS 22.0). Pearson $\chi 2$ analysis was used for the analysis of categorical data. The analysis of the data was carried out in TURCOSA (Turcosa Analytics Ltd. Şti, www.turcosa.com.tr) statistics software. The significance level was accepted as $p<0.05$.

\section{Results}

The records of 286 patients with acute rheumatic fever were analysed. Aspirin treatment was started in 53 of 286 patients (18.5\%) due to arthritis. The mean age of the patients who had used aspirin was $10.7 \pm 2.5$ years. The male to female ratio was 0.8. Echocardiographic evaluation was normal in 7 of the 53 patients $(13.2 \%)$, while the remaining patients had first degree mitral regurgitation and/or aortic regurgitation. Nine of the 53 patients (17\%) who received aspirin treatment were required to change medication due to aspirin-induced hepatotoxicity (naproxen in six patients and ibuprofen in three patients). The mean age of nine patients with aspirin hepatotoxicity was $10.5 \pm 1.9$ years. The remaining 44 patients received a full dose of aspirin. Naproxen or ibuprofen was preferred instead of aspirin in patients who developed aspirin induced hepatotoxicity. No side effects were reported in patients receiving naproxen or ibuprofen. Echocardiographic evaluation of aspirininduced hepatotoxicity revealed seven patients with first-degree mitral regurgitation and two patients with first-degree mitral regurgitation and first-degree aortic regurgitation. Aspirin hepatotoxicity was asymptomatic and there were no accompanying symptoms such as abdominal pain or vomiting. Initially, CRP and ESR values were high in all patients receiving aspirin treatment. However, in patients who developed aspirin hepatotoxicity, serum total/ direct bilirubin, albumin, prothrombin time, INR, ceruloplasmin levels were in the normal range. Viral serology and liver autoantibodies of these patients were also negative.

Elevated AST and ALT values were observed on average $10.9 \pm 6.1$ days when taking aspirin. Of nine patients with elevated aminotransferase levels, three had mild elevation, three had moderate elevation, and the remaining three had severe elevation. In patients who were started on a different non-steroidal antiinflammatory drug after discontinuation of aspirin, aminotransferase elevation decreased to normal limits on average $12.4 \pm 3.9$ days. There was no significant difference in the time of normalization of liver enzymes between patients receiving naproxen or ibuprofen treatment $(p>0.05)$. In patient 6 , aspirin was discontinued and ibuprofen treatment was started because he had a chickenpox infection while taking aspirin. None of the patients with elevated aminotransferase developed druginduced liver failure and no patients required a liver biopsy. Liver enzymes of patients returned to normal after discontinuation of aspirin. Table I shows the laboratory values of patients with aspirin hepatotoxicity.

Hemoglobin levels were below -2SD in four of nine patients with hepatotoxicity (44\%). The mean duration of aspirin use in the hepatotoxic patients who had anemia was $15.7 \pm 6.2$ days. This period was $7.0 \pm 1.4$ days in hepatotoxic patients without anemia $(p=0.02)$. Hemoglobin levels were below -2SD in 12 (27\%) of 44 patients who continued to receive aspirin but did not develop hepatotoxicity. Anemia was detected in $16(30 \%)$ of 53 patients who were started on aspirin treatment. Two of the patients with anemia had folic acid deficiency and one patient had iron deficiency anemia. The etiology of anemia was not investigated in others. However, no statistically significant difference was found between the frequency of anemia in patients with and without hepatotoxicity $(p>0.05)$. Folic acid or iron replacement was applied to those who had folic acid or iron deficiency, and it was determined that the anemia of others improved 
Table I. Laboratory values of patients with aspirin hepatotoxicity.

\begin{tabular}{lccccllll}
\hline Patients & $\begin{array}{c}\text { Age } \\
\text { (years) }\end{array}$ & $\begin{array}{c}\text { CRP } \\
(\mathrm{mg} / \mathrm{L})\end{array}$ & $\begin{array}{c}\mathrm{ESR} \\
(\mathrm{mm} / \mathrm{h})\end{array}$ & $\begin{array}{l}\mathrm{Hb} \\
(\mathrm{g} / \mathrm{dL})\end{array}$ & $\begin{array}{l}\text { Duration to onset } \\
\text { of aspirin induced } \\
\text { hepatotoxicity } \\
\text { (days) }\end{array}$ & $\begin{array}{l}\text { Elevated } \\
\text { AST/ALT } \\
(\mathrm{IU} / \mathrm{L}) \text { levels }\end{array}$ & $\begin{array}{l}\text { Duration to } \\
\text { improvement of } \\
\text { aspirin induced } \\
\text { hepatotoxicity (days) }\end{array}$ & NSAID \\
\hline $1^{*}$ & 15 & 10 & 26 & 11.4 & 17 & $77 / 357$ & 12 & Naproxen \\
$2^{*}$ & 10 & 89 & 79 & 11.2 & 12 & $457 / 461$ & 14 & Naproxen \\
3 & 10 & 70.8 & 74 & 12 & 9 & $1076 / 1573$ & 13 & Ibuprofen \\
$4^{*}$ & 9 & 52 & 104 & 10.8 & 10 & $250 / 260$ & 9 & Naproxen \\
5 & 11 & 34 & 56 & 13.4 & 7 & $107 / 112$ & 14 & Naproxen \\
6 & 8 & 105 & 62 & 13.6 & 5 & $24 / 106$ & 4 & Ibuprofen \\
7 & 10 & 30 & 78 & 14.5 & 7 & $76 / 113$ & 14 & Naproxen \\
8 & 11 & 45 & 51 & 12.8 & 7 & $489 / 402$ & 18 & Ibuprofen \\
$9^{*}$ & 11 & 21 & 45 & 10.6 & 24 & $265 / 356$ & 14 & Naproxen \\
\hline
\end{tabular}

*: Patients with hemoglobin values below -2SD.

$\mathrm{Hb}$ : hemoglobin, ESR: erythrocyte sedimentation rate, NSAID: non-steroidal anti-inflammatory drug, CRP: C-reactive protein

Table II. Comparison of the clinical and laboratory data of the patients with and without hepatotoxicity.

\begin{tabular}{llll}
\hline & $\begin{array}{l}\text { Patients with } \\
\text { hepatotoxicity }(\mathrm{n}=9)\end{array}$ & $\begin{array}{l}\text { Patients without } \\
\text { hepatotoxicity }(\mathrm{n}=44)\end{array}$ & $\mathrm{p}$ \\
\hline Age (years + SD) & $10.5 \pm 1.9$ & $10.8 \pm 2.7$ & $>0.05$ \\
Gender (male/female) & 0.8 & 0.9 & $>0.05$ \\
Mitral regurgitation, $\mathrm{n}$ & 7 & 37 & $>0.05$ \\
Mitral regurgitation and aortic regurgitation, $\mathrm{n}$ & 2 & 0 & $>0.05$ \\
ESR $(\mathrm{mm} / \mathrm{h}$, mean \pm SD) & $63.8 \pm 22.8$ & $65.7 \pm 19.2$ & $>0.05$ \\
CRP $(\mathrm{mg} / \mathrm{L}$, mean \pm SD) & $50.7 \pm 31.8$ & $56.3 \pm 28.7$ & $>0.05$ \\
Frequency of anemia, $\mathrm{n}(\%)$ & $4(44 \%)$ & $12(27 \%)$ & $>0.05$ \\
\hline
\end{tabular}

ESR: erythrocyte sedimentation rate, CRP: C-reactive protein

during the treatment process. Table II shows the comparison of the clinical and laboratory data of the patients with and without hepatotoxicity.

\section{Discussion}

For many years, aspirin has been used as the first-choice non-steroidal anti-inflammatory drug in the treatment of arthritis and mild carditis due to acute rheumatic fever. ${ }^{6}$ Aspirin inhibits prostaglandin synthesis by irreversibly inhibiting cyclooxygenase 1 and thus has an anti-inflammatory effect. Aspirin is a drug with antipyretic, analgesic and anti-inflammatory properties as well as side effects such as dyspepsia, nausea, vomiting, abdominal pain, gastrointestinal ulceration, bleeding and hepatotoxicity. ${ }^{7}$ Aspirin-induced hepatotoxicity has been described for more than 40 years. ${ }^{8}$ Aspirin hepatotoxicity is dosedependent, the higher the dose, the higher the risk of hepatotoxicity, although the mechanism of action of aspirin on the liver is unclear. Hepatotoxicity, which manifests itself as elevated aminotransferase levels, is rapidly recovered by discontinuation of aspirin. ${ }^{9}$ Structural changes in hepatocytes similar to hepatotoxic drugs have been reported in the electron microscopic examination of a liver biopsy of a pediatric patient who developed hepatotoxicity while taking aspirin for acute rheumatic fever. ${ }^{10}$ 
In aspirin hepatotoxicity, aspirin is discontinued and switched to other non-steroidal antiinflammatory drugs such as naproxen, ibuprofen, tolmetin. There is no consensus on which non-steroidal anti-inflammatory drugs will be given first. In the studies, aspirin treatment was primarily adopted as an antiinflammatory treatment in patients with arthritis and mild carditis, however, the use of other non-steroidal anti-inflammatory drugs in case of aspirin intolerance is mentioned..$^{11-13}$ Cetin et al. ${ }^{14}$ reported that they discontinued aspirin and administered naproxen because of aspirin-induced hepatotoxicity in $10 \%$ of children with acute rheumatic fever. In the study of Karademir et al. ${ }^{15}$ they reported that tolmetin can be used effectively and safely in patients who cannot tolerate aspirin treatment. In this study, patients with aspirin hepatotoxicity were treated with naproxen or ibuprofen and no side effects were observed in the patients who were administered either as an alternative to aspirin.

Another serious side effect of aspirin is Reye's syndrome. Aspirin used during viral infection inhibits the fatty acid metabolism by causing mitochondrial damage and ultimately leads to liver failure accompanied by acute encephalopathy. ${ }^{16}$ In our study, aspirin was discontinued and ibuprofen was started in one patient due to chickenpox infection while taking aspirin.

Rasa et al. ${ }^{17}$ found that liver enzyme elevation was $12 \%$ in their study, and they recommended that the liver enzymes be checked every two days after starting aspirin, and weekly followup if there was no elevation. Yilmaz et al. ${ }^{18}$ reported that aspirin hepatotoxicity developed after an average of 11 days from the beginning of aspirin treatment in pediatric patients with acute rheumatic fever, and liver enzymes of patients returned to normal values in an average of 17 days. Olgun et al. ${ }^{19}$ reported that hepatotoxicity was observed approximately 14 days after the start of aspirin treatment, and liver enzymes returned to normal after an average of 16 days after aspirin was discontinued. Karademir et al. ${ }^{15}$ reported that hepatotoxicity developed in the first week in patients using aspirin. In the present study hepatotoxicity secondary to aspirin in the patients was seen on average ten days after the initiation of aspirin treatment. Liver enzymes returned to normal within 12 days after discontinuation of aspirin.

There are limited studies on anemia associated with acute rheumatic fever and $42 \%$ and $62 \%$ of the anemia is mentioned in the studies. ${ }^{17,20}$ Increased plasma volume, hemolytic anemia and decreased erythropoiesis are possible responsible causes of anemia in acute rheumatic fever. ${ }^{17}$ It has been reported that inflammatory proteins such as TNF- $\alpha$ can cause anemia by decreasing erythropoiesis. ${ }^{21}$ In the present study, the frequency of anemia was higher in patients who developed hepatotoxicity than those without hepatotoxicity ( $44 \%$ vs $27 \%$ ), but it was not statistically significant. Hemoglobin values of the patients were not measured before the diagnosis of acute rheumatic fever. The etiology of anemia was investigated in only a few of the patients with anemia at the time of diagnosis, and the necessary vitamin and mineral supplements were given to the patients with folic acid or iron deficiency. However, it was found that the anemia of the patients with anemia improved during the anti-inflammatory treatment process. Most of the patients with arthritis also had mild carditis, and these patients were primarily given steroid therapy. Even though anti-inflammatory therapy may have contributed to the improvement of anemia, but also the improvement of anemia during the treatment period was associated with the improvement in nutrition as a result of increased appetite due to steroid therapy. Hemolytic anemia was not considered in the etiology of anemia because serum bilirubin levels of the patients were within the normal range. Similarly, Rasa et al. ${ }^{17}$ reported that anemia in patients with acute rheumatic fever improved without treatment. Another finding in the present study was that aspirin hepatotoxicity occurred later in patients with anemia than in patients without anemia. In an animal study, it was noted that iron deficiency 
can cause changes in drug metabolism. ${ }^{22}$ In this study, the reason for the late occurrence of aspirin hepatotoxicity in the patients with anemia could not be explained.

The limitations of the study were a small sample size and absence of serum aspirin levels of the patients. However, the fact that no reason was found in the differential diagnosis of acute hepatitis in patients' laboratory tests and the aminotransferase levels returned to normal after aspirin was discontinued supported aspirin hepatotoxicity.

Patients with acute rheumatic fever were retrospectively evaluated in the present study and the rate of development of aspirin-induced hepatotoxicity was $17 \%$. However, $30 \%$ of the cases had anemia. Although aspirin treatment is the first-line anti-inflammatory drug in patients with arthritis and mild carditis in acute rheumatic fever, hepatotoxicity and other systemic side effects should be considered. Naproxen and ibuprofen are safe non-steroidal anti-inflammatory drugs when aspirin-related side effects are observed in the treatment of acute rheumatic fever. In this study, it was determined that the rate of anemia was higher in patients who developed hepatotoxicity. Further studies are needed to determine the relationship between anemia and hepatotoxicity in acute rheumatic fever.

\section{REFERENCES}

1. Eroğlu AG. Update on diagnosis of acute rheumatic fever: 2015 Jones criteria. Turk Pediatri Ars 2016; 51: 1-7.

2. Gewitz MH, Baltimore RS, Tani LY, et al; American Heart Association Committee on Rheumatic Fever, Endocarditis and Kawasaki Disease of the Council on Cardiovascular Disease in the Young. Revision of the Jones criteria for the diagnosis of acute rheumatic fever in the era of Doppler echocardiography: a scientific statement of the American Heart Association. Circulation 2015; 131: 1806-1818.

3. Gitlin N, Grant J. Raised serum transaminase levels in patients with rheumatic fever treated with salicylates. S Afr Med J 1977; 51: 697-698.
4. Uziel Y, Hashkes PJ, Kassem E, Padeh S, Goldman $\mathrm{R}$, Wolach B. The use of naproxen in the treatment of children with rheumatic fever. J Pediatr 2000; 137: 269-271.

5. Vajro P, Maddaluno S, Veropalumbo C. Persistent hypertransaminasemia in asymptomatic children: a stepwise approach. World J Gastroenterol 2013; 19: 2740-2751.

6. Viswanathan V. Acute rheumatic fever. Indian J Rheumatol 2012; 7: 36-43.

7. Baron JA, Senn S, Voelker M, et al. Gastrointestinal adverse effects of short-term aspirin use: a metaanalysis of published rhandomized controlled trials. Drugs R D 2013; 13: 9-16.

8. Prescott LF. Hepatotoxicity of mild analgesics. Br J Clin Pharmacol 1980; 10(Suppl 2): S373-S379.

9. Meunier L, Larrey D. Recent advances in hepatotoxicity of non steroidal anti-inflammatory drugs. Ann Hepatol 2018; 17: 187-191.

10. Iancu T, Elian E. Ultrastructural changes in aspirin hepatotoxicity. Am J Clin Pathol 1976; 66: 570-575.

11. Hashkes PJ, Tauber T, Somekh E, et al; Pediatric Rheumatlogy Study Group of Israel. Naproxen as an alternative to aspirin for the treatment of arthritis of rheumatic fever: a randomized trial. J Pediatr 2003; 143: 399-401.

12. Saxena A, Kumar RK, Gera RP, Radhakrishnan S, Mishra S, Ahmed Z. Working Group on Pediatric Acute Rheumatic Fever and Cardiology Chapter of Indian Academy of Pediatrics. Consensus guidelines on pediatric acute rheumatic fever and rheumatic heart disease. Indian Pediatr 2008; 45: 565-573.

13. Ralph AP, Noonan S, Boardman C, Halkon C, Currie BJ. Prescribing for people with acute rheumatic fever. Aust Prescr 2017; 40: 70-75.

14. Çetin III, Ekici F, Kocabaş A, et al. The efficacy and safety of naproxen in acute rheumatic fever: the comparative results of 11-year experience with acetylsalicylic acid and naproxen. Turk J Pediatr 2016; 58: 473-479.

15. Karademir S, Oğuz D, Senocak F, Ocal B, Karakurt C, Cabuk F. Tolmetin and salicylate therapy in acute rheumatic fever: comparison of clinical efficacy and side-effects. Pediatr Int 2003; 45: 676-679.

16. Uppala R, Dudiak B, Beck ME, et al. Aspirin increases mitochondrial fatty acid oxidation. Biochem Biophys Res Commun 2017; 482: 346-351.

17. Rasa M, Garcia A, Yamaga K, et al. Hematologic and hepatic anomalies in pediatric acute rheumatic fever. Ann Pediatr Res 2018; 2: 1-5. 
18. Yılmaz M, Gürses D. Akut romatizmal ateş tedavisinde aspirine bağlı hepatotoksisitenin değerlendirilmesi. Pamukkale Tıp Dergisi 2020; 13: 268-274.

19. Olgun H, Bulgan M, Ceviz N, Yolcu C, Sahin IO, Laloglu F. 51st Annual Meeting of the Association for European Paediatric and Congenital Cardiology (AEPC). Incidence of aspirin-related hepatotoxicity in pediatric cases with acute rheumatic fever. Cardiol Young 2017; 27(Suppl 2): S73-S74.

20. Bitar FF, Hayek P, Obeid M, Gharzeddine W, Mikati M, Dbaibo GS. Rheumatic fever in chidren: a 15-year experience in a developing country. Pediatr Cardiol 2000; 21: 119-122.
21. Jacobs-Helber SM, Roh KH, Bailey D, et al. Tumor necrosis factor-alpha expressed constitutively in erythroid cells or induced by erythropoietin has negative and stimulatory roles in normal erythropoiesis and erythroleukemia. Blood 2003; 15: 524-531.

22. Dhur A, Galan P, Hercberg S. Effects of different degrees of iron deficiency on cytochrome P450 complex and pentose phosphate pathway dehydrogenases in the rat. J Nutr 1989; 119: 40-47. 